\title{
Plaguicidas para el manejo del ácaro blanco (Polyphagotarsonemus latus, Banks.) (Acarina; Tarsonemidae), en pimiento dulce (Capsicum annuum L.), bajo condiciones protegidas en Nicaragua
}

\section{Pesticides for the management of the broad mite (Polyphagotarsonemus latus, Banks.) (Acarina; Tarsonemi- dae), in green pepper (Capsicum annuum L.), under protected conditions in Nicaragua}

\author{
${ }^{1}$ Darwin Raudez-Centeno, ${ }^{2}$ Edgardo Jiménez-Martínez \\ 'Instituto Nicaragüense de Tecnología Agropecuaria (INTA), Investigador-MIP, E-mail: darwincenteno19@yahoo.es / \\ Tel: (505) 5706-4194 \\ ${ }^{2}$ Profesor titular, ORCID: https://orcid.org/0000-0003-1086-7380 Universidad Nacional Agraria, edgardo.jimenez@ci.una.edu.ni / Tel: (505)
} 2263-2609

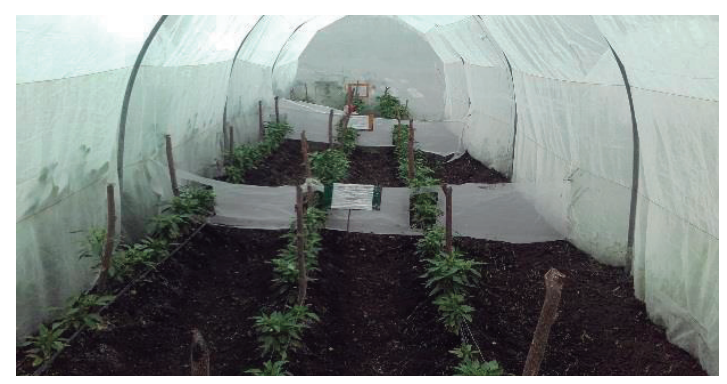

\section{RESUMEN}

El ácaro blanco Polyphagotarsonemus latus (Banks) es la principal plaga causante de pérdidas económicas en el pimiento dulce en San Isidro, Matagalpa. En base a esta problemática se realizó un estudio, con el objetivo de evaluar pesticidas e identificar el tratamiento más efectivo en el manejo de ácaro blanco en chiltoma variedad tres cantos bajo condiciones protegidas. El ensayo se realizó en el período de mayo a julio del 2016. Los tratamientos evaluados fueron Ajo+chile+jabón, Caldo sulfocalcico, Oberón ${ }^{\circledR}$ y Abamectina ${ }^{\circledR}$ comparándolo con el testigo que fue solamente agua, las variables evaluadas fueron número de ácaro blanco por planta, incidencia, severidad, altura y rendimiento en $\left(\mathrm{kg} \mathrm{ha}^{-1}\right)$ y algunas variables económicas como análisis del presupuesto parcial, análisis de dominancia y análisis de la tasa de retorno marginal el diseño experimental fue de bloque completo al azar (BCA) con tres repeticiones. Los resultados demuestran que el tratamiento Oberón ${ }^{\circledR}$ y Ajo+chile+jabón presentan el mejor efecto sobre el control de ácaro blanco al obtener menores individuos por planta y la menor incidencia, al igual el tratamiento Ajo+chile+jabón y el tratamiento Oberón ${ }^{\circledR}$ presentaron los menores porcentajes de severidad, no se presentó diferencia entre las alturas de los tratamientos evaluados, los mejores rendimientos comerciales los obtuvieron los tratamientos Oberón ${ }^{\circledR}$ y el tratamiento Ajo+chile+jabón, la mejor tasa de retorno marginal fue lograda por el tratamiento Ajo+chile+jabón, seguido del tratamiento Oberón $\mathbb{R}$

Palabras clave: incidencia, Nicaragua, rendimiento, severidad.
Recibido: 11 de octubre del 2017 Aceptado: 2 de marzo del 2018

\begin{abstract}
The broad mite Polyphagotarsonemus latus (Banks) is the main pest causing economic losses in green pepper in San Isidro, Matagalpa. Based upon this problem, a study was carried out with the objective of evaluating pesticides and identifying the most effective treatment for the management of the broad mite in the green pepper variety "Tres cantos" under protected conditions The trial was carried out from May to July 2016. Evaluated treatments were, garlic+chili+soap, Sulfo-calcium broth, Oberon ${ }^{\circledR}$ and Abamectina ${ }^{\circledR}$, compared to the control treatment that was only water use, the evaluated variables were, broad mite number per $\left(\mathrm{kg} \mathrm{ha}^{-1}\right)$ and some economic variables such as partial budget analysis, dominance analysis and marginal rate of return analysis, the experimental design was a randomized complete block (BCA) with three replicates. Results show that the Oberón ${ }^{\circledR}$ and garlic + chili + soap treatment, presented the best effect on the management of the broad mite, obtaining smaller number of individuals per plant, and the lower incidence, as well these two treatment presented, the highest commercial yields, the best marginal rate of return was achieved by the treatment garlic+Chili+soap, followed by Oberón ${ }^{\circledR}$.
\end{abstract}

Key words: Incidence, pest management, mite, Nicaragua. 


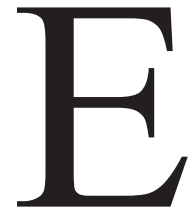

1 pimiento dulce (Capsicum annum, L.), es una planta que pertenece a la familia de las solanáceas y es originario de regiones tropicales específicamente de Bolivia y Perú (INTA, 2004). De estos países se difundió por la mayor parte del continente americano, adaptándose a las diferentes regiones como el sur de Brasil, México y luego el resto del mundo (CATIE, 1993).

El fruto de esta hortaliza es muy importante para el consumo humano por sus cualidades nutritivas, con alto contenido de carotenos, vitaminas A y $\mathrm{C}$ además de minerales. Su sabor agradable y estimulante hacen que esta hortaliza sea valiosa y esencial en la dieta alimenticia de los seres humanos (Bolaños, 1998).

En la actualidad, el pimiento dulce se cultiva en la mayoría de los países tropicales y subtropicales del mundo siendo China, México, Turquía, Estados Unidos y España los principales países que producen este rubro (FAO, 2004).

El uso incorrecto de los productos químicos sintéticos ha generado desequilibrio en el agroecosistema, provocando que organismos que antes se consideraban como plagas secundarias se conviertan en plagas primarias (González y Obregón, 2007).

Los insecticidas botánicos son preparados naturales a partir de extractos vegetales, el resultado obtenido al aplicarlos son muerte de insectos por envenenamiento (Chile, Nim), inhiben la alimentación (alcaloides del tabaco), muerte por inanición, antagonismo de hormonas de insectos (Nim); (Jiménez-Martínez y Varela, 2013)

El chile contiene una sustancia de pungencia elevada llamada capsicina que al ser aplicada Sobre los insectos plaga genera una sensación de ardor en todo su cuerpo, por lo cual los insectos dejan de alimentarse, huyen del lugar y mueren (Jiménez-Martínez y Varela, 2013).

El ajo (Allium sativum, L.) contiene compuestos de azufre (tiosulfatos) los cuales sobre excitan el sistema nervioso de los insectos y ácaros produciendo irritación, desorientación y repelencia (Jiménez-Martínez y Varela, 2013).

Según King y Saunders, (1984) El pimiento dulce es afectada por un sin número de plagas de artrópodos como insectos y ácaros así como enfermedades de tipo infeccioso, entre las plagas más comunes se encuentran los minadores de la hoja (Lyriomiza trifolli, Burgués) picudo del fruto (Anthonomus eugenni, Cano) y algunas especies de ácaros en especial (P. latus) y (Tetranichus urticae, Koch).

Según el CENAGRO (2011) en Nicaragua el cultivo de el pimiento dulce se siembra en los 17 departamen- tos con un área total de $2218 \mathrm{mz}$, la cual en Matagalpa se siembra un $18 \%$ con un total de $402.98 \mathrm{mz}$, seguido de Managua que siembra $14 \%$ con un total de $315.8 \mathrm{mz}$ de igual forma Jinotega siembra un total de $302.17 \mathrm{mz}$ con un $13.6 \%$ de la producción total. Asi como en otras regiones donde se cultivan a menor escala como Ocotal, Somoto, Estelí, Jinotega, Boaco, Granada, Juigalpa y Masaya (INTA, 2004).

El ácaro blanco es una plaga que se ha convertido en un severo problema fitosanitario y socioeconómico para los productores de chiltoma debido a su difícil control y resistencia a plaguicidas químicos, llegando a producir grandes pérdidas debido a que los ácaros se agrupan en colonias en el envés de las hojas y prefieren los terminales para su desarrollo y alimentación (Sevilla y Rodríguez, 2009).

En los últimos cuatro años en la zona de San Isidro el cultivo de chiltoma es muy afectada por el ácaro blanco ocasionando pérdidas de hasta el cien por ciento de la producción, ante esta situación pretendemos con esta investigación conocer si algunos plaguicidas son eficaces para el manejo de esta plaga bajo condiciones protegidas y con estos resultados poder brindar información sobre la efectividad de los plaguicidas evaluados que sean económicamente rentables pero además que sean amigables con el medio ambiente.

\section{MATERIALES Y MÉTODOS}

Ubicación del área de estudio. Esta investigación se realizó de mayo a julio del 2016, en el Tecnológico Nacional IPADEN ubicado en el municipio de San Isidro con latitud de $12^{\circ} 55^{\prime}$ y longitud $86^{\circ} 11^{\prime}$ (CENAGRO, 2011).

Diseño experimental. El ensayo se estableció como un experimento en diseño de bloques completo al azar (BCA) con tres repeticiones y cinco tratamientos, el tamaño de la parcela correspondió a $4 \mathrm{~m}$ de largo por 2,4 $\mathrm{m}$ de ancho para un área de $9,6 \mathrm{~m}^{2}$ por cada tratamiento formando un área de $48 \mathrm{~m}^{2}$ por cada repetición, siendo un área total del ensayo de $144 \mathrm{~m}^{2}$.

Tratamientos evaluados. Tratamiento 1: chile (Capsicum annum, L.) Fam. Solanáceas + Ajo (Allium sativum, L.) Amaryllidaceae + Jabón (detergente xedex). El chile contiene una sustancia de pungencia elevada llamada capsicina que al ser aplicada sobre los insectos plaga genera una sensación de ardor en todo su cuerpo, por lo cual los insectos dejan de alimentarse, huyen del lugar y mueren (Jiménez-Martínez y Barberena, 2013). El ajo contiene compuestos de azufre (tiosulfatos) los cuales sobre excitan 
el sistema nervioso de los insectos y ácaros produciendo irritación, desorientación y repelencia (Jiménez-Martínez y Jirón, 2013). El detergente actúa como adherente al follaje, cuando entra en contacto con los tejidos grasosos del ácaro lo mata por deshidratación (Rayo y Mena, 2015). La dosis utilizada fue de 100 gramos de chile, 28 gramos de detergente y una cabeza de ajo, molido y disuelto en un litro de agua, con 24 horas de reposo, por bomba de 20 litros (Jiménez-Martínez e Izaguirre 2013).

Tratamiento 2: Caldo Sulfocálcico. Es un producto químico que se obtiene de la mezcla de azufre, cal y agua, su modo de penetración es por contacto. El tratamiento se aplicó por aspersión al follaje una vez que se encontró un promedio del ácaro por hoja, con dosis de $500 \mathrm{cc}$ por 18 litros de agua (Sevilla y Rodríguez, 2009).

Tratamiento 3: Oberón ${ }^{\circledR}$. Insecticida, acaricida, el ingrediente activo es el spiromesifen, penetra por contacto, su modo de acción la realiza al inhibir la síntesis de lípidos, causando intoxicación al ácaro (Jiménez-Martínez et al., 2013)

Tratamiento 4: Abamectina ${ }^{\circledR}(1.8 \mathrm{EC}+$ Imidacloprit + Fipronil). Es un insecticida de origen natural (aislados de productos de la fermentación del microrganismo del suelo (Streptomyces avermitilis, Kim y Goodfellow), perteneciente al grupo químico de las lactonas macrocíclicas. Se caracteriza por ser de amplio espectro, largo efecto residual y actuar por contacto, ingestión y de forma translimitar este insecticida actúa sobre los activadores de los canales de cloro, como el canal de glutamato de cloro (GluCls) y el ácido gamma-amino-butírico que son neurotransmisores inhibitorios importantes en el sistema nervioso de los insectos, el insecto se paraliza y la actividad visible de éste como alimentarse y oviponer se detiene y dentro de poco tiempo muere (Santolaria, 2013).

Testigo. En este tratamiento se aplicó agua solamente.

Descripción del estudio. El estudio consistió en el establecimiento de un semillero bajo condiciones de micro invernadero, se utilizó la variedad de chiltoma tres cantos, la cuál se caracteriza por tener forma de tres cantos con un color de fruto verde en estado pinto y rojo en estado maduro un largo de fruto de 10 a $12 \mathrm{~cm}$ con una adaptabilidad ambiental hasta los $1300 \mathrm{msnm}$ recomendada para todos los municipios que producen hortalizas en el país (INTA, 2005), se utilizaron 12 bandejas de polietileno de 108 celdas pasadas por agua caliente para su desinfección, el sustrato utilizado fue a base de cascarilla de arroz carbonizado + lombrihumus en proporción 1: 1.El pimiento dulce se trasplantó en microtunel elaborados a base de tubos de 1 pulgada galvanizados y soldados con varillas de soldar 360, cubiertos con malla agribon de 50 mesh o sea 50 agujeros de $(0.3 \times 0.4 \mathrm{~cm})$, por pulgada.

La preparación de suelo se realizó de forma mecanizada utilizando un pase de romeplow, dos pases de grada y un pase de niveladora se realizó desinfección con cal a razón de $4500 \mathrm{~kg} \mathrm{ha}^{-1}$, la distancia entre cada surco utilizada fue de $0.8 \mathrm{~m} \times 0.35 \mathrm{~m}$ entre planta, en total se prepararon 9 surcos, 57 plantas por cada surco, 171 plantas por repetición y 27 plantas por tratamiento. Se tendieron cintas de riego por goteo en cada uno de los camellones para suministrar agua a las plántulas durante el ensayo.

El trasplante se realizó de manera manual estableciendo una plántula por cada orificio hecho en el camellón al momento se aplicó Fertilizante completo $(18-46-0) \circledR$ diluido al drench a razón de $32 \mathrm{~kg} \mathrm{ha}^{-1}$ seguido de previcur ${ }^{\circledR} 0.491$ ha $^{-1}$ y carbendazim ${ }^{\circledR} 0.71$ ha $^{-1}$ ambos aplicados en bomba de mochila de 20 litros para evitar daños por (Fusarium oxisporium, Franz.) y (Phytium aphanidermatum, Edson.).

Las fertilizaciones realizadas se hicieron de manera basal diluidas desde un día antes a razón de $332 \mathrm{~kg} \mathrm{ha}^{-1}$ de fertilizante completo $(0-0-60) \circledast, 434 \mathrm{~kg} \mathrm{ha}^{-1}$ de fertilizante completo $(18-46-0) \AA, 177 \mathrm{~kg} \mathrm{ha}^{-1}$ de urea $46 \%$ (46-0-0)®, aplicado semanalmente de igual forma se aplicaron fertilizantes foliares como biofertilizantes enriquecidos con micro elementos (B-Ca-Mg-Zn-P) a razón de 4.21 ha $^{-1}$ rotándolos con Byfolan Forte ${ }^{\circledR}$ en dosis de 1.41 ha $^{-1}$ y Oligomix $+\mathrm{Ca}{ }^{\circledR}$ a razón de 0.561 ha $^{-1}$ de igual manera aplicados semanalmente.

Para el manejo de malezas se realizaron aplicaciones de Basta ${ }^{\circledR}$ (Glifusonato de Amonio) a razón de 2.1 1/ha-1 en el caso de Coyolillo (Cyperus rotundus L.) se manejó de manera manual.

En el caso de las enfermedades como (Alternaría solanii, Cooke), (Phytophthora infestan Mont) y (Cercospora capsici, Heald \& F.A. Wolf). Se aplicaron fungicidas como Amistar ${ }^{\circledR}$ a razón de $0.16 \mathrm{~kg} \mathrm{ha}^{-1}$ y Mancozeb ${ }^{\circledR}$ a razón de $1.4 \mathrm{~kg} \mathrm{ha}^{-1}$ de igual forma se utilizaron productos como Clorotalonil ${ }^{\circledR}$ a 0.841 ha $^{-1} \mathrm{y}$ Carbendazim ${ }^{\circledR} 0.71$ ha $^{-1}$.

La cosecha se realizó de manera manual pesando el número de frutos obtenidos por cada tratamiento con pesa de reloj de 20 libras marca Chunter ${ }^{\circledR}$.

Los muestreos se realizaron dos veces por semana desde los $10 \mathrm{ddt}$ hasta los $51 \mathrm{ddt}$ por la mañana utilizando lupa 30xFH77 marca Pointer ${ }^{\circledR}$ se muestrearon 10 plantas por cada tratamiento, para un total de 50 plantas por repetición y 150 plantas en todo el experimento muestreando solo los brotes tiernos. (Jimenez-Martínez et al., 2013). 
Las aplicaciones de los tratamientos se realizaron cuando se encontró un nivel poblacional promedio de un ácaro blanco por planta (Jimenez-Martínez et al., 2013) se utilizó bomba de mochila Matabi ${ }^{\circledR}$ con capacidad para 20 litros de agua.

\section{Variables evaluadas}

Fluctuación poblacional del ácaro blanco. Esta variable se comenzó a tomar desde los 15 días después del trasplante realizando observaciones dos veces por semana, por la mañana hasta los 51 días después del trasplante, donde se muestrearon todos los brotes nuevos de las plantas específicamente el envés de las hojas, lugar donde se encuentran más frecuente el ácaro (Ochoa et al., 1991).

Porcentaje de incidencia y severidad del daño de ácaro blanco. La incidencia es la relación de las plantas que presentan síntomas de daño del ácaro blanco con relación al número total de las plantas muestreadas multiplicadas por cien, la severidad es el porcentaje de tejido visible dañado o afectado de una planta con relación al total evaluado. Para diferenciar una planta sana de una enferma esta se realiza a través de la observación del síntoma característico que presentan las hojas de las plantas afectadas por el ácaro, como es la clorosis y deformación de las hojas y ramas (Rayo y Mena, 2015).

Para determinar el porcentaje de incidencia y severidad del daño del ácaro se realizó toma de datos desde los 15 días hasta los 51 días después del trasplante utilizando la fórmula para determinar incidencia, que consiste en dividir el número de plantas con acaro blanco, por el muero de plantas muestreadas, multiplicado por cien.

Para determinar el grado de severidad ocasionado por el ácaro blanco se usó la escala de severidad utilizada en estudios anteriores por (Jiménez-Martínez, 2013), la cual define grado 0 , cuando no hay presencia de síntomas, grado 1, débil encrespado hacia arriba en la lámina foliar de hojas nuevas y brotes nuevos, grado 2, ondulación en hojas nuevas y viejas, grado 3, encrespado hacia arriba y deformación en la nervadura central en forma de zigzag y grado 4, hojas severamente dañadas, caída de las mismas $\mathrm{y}$ aborto de frutos, enanismo en las plantas.

Para obtener el grado porcentual de la severidad se utilizó la fórmula general planteada por Vanderplank, (1963), según la cual, el porcentaje de severidad es igual a la sumatoria de severidad en plantas muestreadas dividido por el producto del número de plantas muestreadas por el valor máximo de la escala, todo ello multiplicado por cien.
Rendimiento de pimiento dulce. Para obtener los datos de rendimiento por hectárea se efectuaron seis cortes cada 15 días se cosecharon frutos verdes y maduros, se pesó el total de frutos cosechados por cada parcela de los tratamientos evaluados.

Análisis económico. Se determinó la rentabilidad de los tratamientos sometiendo los datos a un análisis económico de las variables agronómicas mediante un análisis de presupuesto parcial a través de la metodología del CIMMYT, (1988), el cual incluye, además, el análisis de dominancia $(\mathrm{AD})$ y la determinación de la tasa marginal de retorno (TMR), acorde a la metodología propuesta por CIMMYT (1988).

Análisis estadísticos de las variables. Se construyó una base de datos en Microsoft Excel a través del cual se analizaron las variables en estudio, se realizó el análisis de varianza para probar la hipótesis de igualdad entre medias de tratamientos se utilizó la prueba de separación de medias de rangos múltiples de Tukey al 5\% de significancia $(p \leq 0.05)$, para lo cual se utilizaron los paquetes estadísticos INFOSTAT (Di Rienzo et al., 2013) e INFOGEN (Balzarini y Di Rienzo, 2012).

\section{RESULTADOS Y DISCUSIÓN}

Fluctuación poblacional de ácaro blanco. Las fluctuaciones más altas de ácaro blanco se presentaron a los 17 y 31 ddt a los 17 ddt los tratamientos Oberón $\AA$, Abamectina ${ }^{\circledR}$ presentaron las poblaciones más bajas con 2.6 y 4.9 ácaros por planta, a los $24 \mathrm{ddt}$ los tratamientos Oberón ${ }^{\circledR}, A j o+$ chile+jabóny Abamectina ${ }^{\circledR}$ presentaron las poblaciones más bajas con 2.3, 5.7 y 6.3 ácaros por planta, a los 31 ddt los tratamientos Oberón ${ }^{\circledR}$, Ajo+chile+jabón fueron los que presentaron las poblaciones más bajas con 1 y 4 ácaros por planta en el análisis de diferencia de medias según Tukey demuestra que existe diferencia significativa $(p \leq 0.001)$ entre los tratamientos evaluados obteniendo que el menor número de ácaros lo presentó el tratamiento químico Oberón ${ }^{\circledR}$, con 1.23 ácaros por planta, seguido del tratamiento Abamectina ${ }^{\circledR}$, con 5.63 ácaros por planta y el tratamiento Ajo+chile+jabón con 5.71 ácaros no encontrándose diferencia significativa entre ambos, la mayor presencia de ácaro por planta se refleja en el testigo (agua) con 9.46 ácaros por planta (cuadro 1). Según Ochoa et al., (1991) el ácaro blanco de El pimiento dulce se encuentra comúnmente en el envés de las hojas jóvenes donde provoca el mayor daño y logra establecer grandes poblaciones. 
Según Rodríguez et al., (2012) las aspersiones con Dicofol ${ }^{\circledR} 18.5$ C.E, y Abamectina ${ }^{\circledR}$ 1.8 E.C, disminuyen las poblaciones del ácaro blanco en chiltoma, pero tiende a incrementar al final del ciclo del cultivo.

Porcentaje de incidencia de $\boldsymbol{P}$. latus. Desde los 10 días después del trasplante se presentaron porcentajes de incidencia en plantas en los tratamientos evaluados a los 45 ddt los tratamientos Oberón ${ }^{\circledR}$, Abamectina ${ }^{\circledR}$, y Ajo + chile + jabón presentaron los porcentajes de incidencia más bajos con $0,46.7$ y $56.6 \%$ respectivamente. Los tratamientos caldo sulfocálcico y el testigo a los 17 días después del trasplante presentan los porcentajes de incidencia más altos con 98 y $96.7 \%$ en este mismo período el tratamiento Oberón ${ }^{\circledR}$, Abamectina ${ }^{\circledR}$, y Ajo+chile+jabón presentaron los porcentajes de incidencia más bajos con $26.6,50$ y $56.6 \%$. respectivamente. En el análisis de diferencia de medias realizado a la variable incidencia según Tukey al $5 \%$ demuestra que existe diferencia significativa $(p \leq 0.001)$ entre los tratamientos evaluados, obteniendo que el tratamiento Oberón ${ }^{\circledR}$ fue el que presentó la menor media del porcentaje de incidencia con $11.06 \%$ de afectación, seguido del tratamiento Abamectina ${ }^{\circledR}$ y Ajo+chile+jabón, los cuales se comportaron de manera similar con 53.6 y $54.7 \%$ de incidencia causada por ácaro blanco respectivamente (cuadro 1). Debido a sus características etológicas, biológicas y ecológicas, $P$. latus, es una plaga de difícil control a través de los métodos y medios más utilizados en la protección de los cultivos (Rodríguez et al., 2013; Rodríguez et al., 2015).

Severidad del daño ocasionado por ácaro blanco. Los tratamientos Oberón ${ }^{\circledR}$, Ajo+chile+jabón y Abamectina ${ }^{\circledR}$ presentaron los porcentajes de severidad más bajos con 0 , 12.5 y $17.5 \%$ de afectación a los $53 \mathrm{ddt}$, en este mismo periodo los tratamientos caldo sulfocálcico y el testigo presentaron los porcentajes de severidad mas altos con 42 y $87.5 \%$ respectivamente. El análisis de diferencia de medias según Tukey al 5\% demuestra que existe diferencia significativa $(\mathrm{P} \leq 0.001)$ entre los tratamientos evaluados obteniendo que el tratamiento Oberón ${ }^{\circledR}, A j o+$ chile + jabón y Abamectina ${ }^{\circledR}$ presentan los porcentajes de severidad más bajos con medias de $2.66,15.63$ y $19.03 \%$ (cuadro 1 ). Los tratamientos que presentaron los porcentajes de severidad más altos fueron el testigo y el caldo sulfocálcico con 58.03 y $31.93 \%$ De Coss-Romero y Pena, (1998) señalan que los ácaros fitophagos como el ácaro blanco, por las características de su parte orales, son menos capaces de penetrar en los tejidos lignificados lo que es probable que exista la mayor severidad en los rebrotes tiernos.

Comparación del rendimiento total obtenido en $(\mathrm{kg}$ $\left.\mathbf{h a}^{-1}\right)$. La comparación de los rendimientos entre los tratamientos demuestra que el tratamiento con mayor rendimiento fue el Oberón ${ }^{\circledR}$ con $33545 \mathrm{~kg} \mathrm{ha}^{-1}$ seguido del tratamiento Ajo+chile+jabón que logro un rendimiento

Cuadro 1. Ácaro blanco por planta, porcentaje de incidencia y severidad del daño por tratamiento, mayo a julio 2016

\begin{tabular}{|c|c|c|c|}
\hline Tratamientos & $\begin{array}{l}\text { ácaros/planta } \\
\text { Medias } \pm \text { ES }\end{array}$ & $\begin{array}{l}\% \text { incidencia } \\
\text { Medias } \pm \text { ES }\end{array}$ & $\begin{array}{l}\% \text { Severidad } \\
\text { Medias } \pm \text { ES }\end{array}$ \\
\hline Caldo sulfocálcico & $9.10 \pm 0.45 \mathrm{a}$ & $88.06 \pm 3.17 \mathrm{a}$ & $58.03 \pm 0.50 \mathrm{a}$ \\
\hline Testigo & $9.46 \pm 0.28 \mathrm{a}$ & $91.66 \pm 2.17 \mathrm{a}$ & $31.93 \pm 2.31 \mathrm{~b}$ \\
\hline Ajo + chile + jabon & $5.76 \pm 1.85 b$ & $54.73 \pm 15.66 b$ & $15.63 \pm 4.48 \mathrm{c}$ \\
\hline Abamectina ${ }^{\circledR}$ & $5.63 \pm 0.30 \mathrm{~b}$ & $53.63 \pm 5.09 \mathrm{~b}$ & $19.03 \pm 2.40 \mathrm{c}$ \\
\hline Oberón ${ }^{\circledR}$ & $1.23 \pm 0.45 \mathrm{c}$ & $11.66 \pm 4.44 \mathrm{c}$ & $2.66 \pm 1.15 \mathrm{~d}$ \\
\hline $\mathrm{N}$ & 93 & 899 & 381 \\
\hline $\mathrm{SD} ; \mathrm{CV}$ & $3.17 ; 13.01$ & $30.70 ; 51.20$ & $19.54 ; 10.85$ \\
\hline$(\mathrm{F} ; d f ; P)$ & $10.05 ; 8 ; 0.001$ & $942 ; 8 ; 0.001$ & $381.9 ; 8 ; 0.001$ \\
\hline
\end{tabular}

Medias con distintas letras son estadísticamente diferentes según Tukey $\infty 0.05$

$\mathrm{ES}=$ Error estándar, $\mathrm{CV}=$ Coeficiente de Variación, $\mathrm{SD}=$ Desviación Estándar, $\mathrm{N}=$ Número de datos utilizados en el análisis, $\mathrm{F}=$ Fisher calculado, $d f=$ Grados de libertad del error, $P=$ Probabilidad según Tukey.

de $31594 \mathrm{~kg} \mathrm{ha}^{-1}$, en cambio los demás tratamientos, Caldo sulfocálcico, Abamectina ${ }^{\circledR}$ y el testigo obtuvieron los rendimientos más bajos con 2657521750 y 19043 $\mathrm{kg} \mathrm{ha}^{-1}$ respectivamente. El rendimiento es el resultado del efecto combinado de muchos factores tanto genéticos como ecológicos, así como la interacción del genotipo con el medio ambiente incluyendo dentro de este último la influencia de la actividad humana mediante el manejo que se le da a la plantación (González y Berbís, 1983).

\section{Comparación económica entre los tratamientos} Presupuesto parcial. Según el CYMMYT (1988) el paso inicial para efectuar un análisis económico de los ensayos es calcular los costos que varían en cada tratamiento. Para determinar los costos y beneficios de los tratamientos evaluados, estos se analizaron con el método del presupuesto parcial. 
EL análisis de presupuesto parcial realizado según la metodología CIMMYT (1988) nos muestra que los mayores costos variables se presentan en los tratamientos Oberón ${ }^{\circledR}$ y Abamectina ${ }^{\circledR}$ con 322.64 y 262.24 USD ha $^{-1}$ y los que presentaron los menores costos variables fueron Caldo Sulfocálcico y Ajo+chile+jabón con 106.9 y 97.09 USD ha $^{-1}$ seguido del testigo con 38.94 USD ha $^{-1}$ respectivamente. tratamiento que presentó los menores beneficios fue el testigo con $6254.56 \mathrm{USD} \mathrm{ha}^{-1}$.

Análisis de dominancia. El análisis de dominancia define el dominio de aquellos tratamientos en cuanto a beneficio netos y costos variables se refiere (CIMMYT 1988).

El análisis de dominancia nos demuestra que existen dos tratamientos que se comportan como

Cuadro 2. Presupuesto parcial de tratamientos plaguicidas para el manejo del ácaro blanco en pimiento dulce bajo condiciones protegidas en Nicaragua

\begin{tabular}{|c|c|c|c|c|c|}
\hline Tratamiento & Ajo+chile+jabón & Caldo Sulfocálcico & Oberón ${ }^{\circledR}$ & Abamectina ${ }^{\circledR}$ & Testigo \\
\hline Rendimiento (kg ha $\left.{ }^{-1}\right)$ & 31594.00 & 26575.00 & 33545.00 & 21750.00 & 19043.00 \\
\hline Rendimiento ajustado $(10 \%)$ & 28434.00 & 23917.00 & 30191.00 & 19575.00 & 17138.00 \\
\hline Precio de campo (USD) & 0.45 & 0.45 & 0.45 & 0.45 & 0.45 .00 \\
\hline Ingreso bruto (USD) & 12796.00 & 10763.00 & 13586.00 & 8809.00 & 7712.00 \\
\hline \multicolumn{6}{|c|}{ Costos que varían (CV) } \\
\hline Costos de los insecticidas (USD) & 58.15 & 67.96 & 283.7 & 223.3 & 0 \\
\hline Costo de aplicación (USD/d/h/ha ${ }^{-1}$ ) & 38.94 & 38.94 & 38.94 & 38.94 & 38.94 \\
\hline Costo total de la aplicación & 97.09 & 106.90 & 322.64 & 262.24 & 38.94 \\
\hline \multicolumn{6}{|c|}{ Costos fijos (CF) } \\
\hline Depreciación de invernadero (ciclo) & 7.09 & 7.09 & 7.09 & 7.09 & 7.09 \\
\hline Depreciación de aspersor (Ciclo) & 4.15 & 4.15 & 4.15 & 4.15 & 4.15 \\
\hline Depreciación de bandejas(ciclo) & 27.50 & 27.50 & 27.50 & 27.50 & 27.50 \\
\hline Depreciación de molino de mano & 0.60 & 0.60 & 0.60 & 0.60 & 0.60 \\
\hline Depreciación de azadón(ciclo) & 0.64 & 0.64 & 0.64 & 0.64 & 0.64 \\
\hline Costo de semilla (USD ha-1) & 29.07 & 29.07 & 29.07 & 29.07 & 29.07 \\
\hline Costos de fertilizantes & 776.21 & 776.21 & 776.21 & 776.21 & 776.21 \\
\hline Costo total mano de obra ha ${ }^{-1}$ & 573.66 & 573.66 & 573.66 & 573.66 & 573.66 \\
\hline Total de costos fijos (USD ha-1) & 1418.92 & 1418.92 & 1418.92 & 1418.92 & 1418.92 \\
\hline Total costos variables (USD ha-1) & 97.09 & 106.90 & 322.64 & 262.24 & 38.94 \\
\hline Total costos de producción (USD ha-1) & 1516.01 & 1525.82 & 1741.56 & 1681.16 & 1457.86 \\
\hline Beneficio neto (USD ha ${ }^{-1}$ ) & 11279.56 & 9237.06 & 11844.62 & 7127.59 & 6254.56 \\
\hline
\end{tabular}

Precio oficial del dólar a la fecha (septiembre, 2016), C\$28.89 Según BCN. Precio del pimiento dulce en el campo al momento de la cosecha 0.45 USD por cada kilogramo.

El mejor beneficio neto se obtiene con el tratamiento Oberón ${ }^{\circledR}$ con $11844.62 \mathrm{USD} \mathrm{ha}^{-1}$ por efecto de un mayor rendimiento por hectárea seguido del tratamiento Ajo+chile+jabón con 11279.56 USD ha $^{-1}$ el dominados, estos son, el caldo sulfocálcico y el tratamiento Abamectina ${ }^{\circledR}$ esto es debido a que presentan altos costos variables y bajos beneficios netos, los tratamientos testigo, Ajo+chile+jabón y Oberón ${ }^{\circledR}$ resultaron ser no dominado, 
Cuadro 3. Análisis de Dominancia de tratamientos plaguicidas para el manejo del ácaro blanco en pimiento dulce bajo condiciones protegidas en Nicaragua

\begin{tabular}{lccc}
\hline \multicolumn{1}{c}{ Tratamiento } & Costo variable US $\$ \mathrm{ha}^{-1}$ & ${\text { Beneficio neto US } \$ / \mathrm{ha}^{-1}}$ & Dominancia \\
\hline Testigo & 38.9 & 6255 & $\mathrm{ND}$ \\
Ajo+chile+jabón & 97.1 & 11280 & $\mathrm{ND}$ \\
Caldo Sulfocálcico & 106.9 & 9237 & $\mathrm{D}$ \\
Abamectina ${ }^{\circledR}$ & 262.2 & 7128 & $\mathrm{D}$ \\
Oberón ${ }^{\circledR}$ & 322.6 & 11845 & $\mathrm{ND}$ \\
\hline
\end{tabular}

ND: No Dominado D: Dominado.

por lo tanto, serán los únicos a tomar en cuenta para realizar el análisis de la tasa de retorno marginal.

Análisis de la tasa de retorno marginal (TRM). El análisis de la tasa de retorno marginal es la decisión de ganancia al momento de probar una tecnología comparada con otra que presente mayor rentabilidad que la usada anteriormente, según el CIMMYT (1988), la tasa de retorno marginal mínima aceptable por el agricultor es de 50 y $100 \%$ lo que indica que su inversión disminuya cuando decide cambiarse de una tecnología a otra en ese rango. El análisis de tasa de retorno marginal refleja que para el manejo del ácaro blanco el mejor tratamiento es el Ajo+chile+jabón ya que por cada dólar invertido el productor obtiene una tasa de retorno marginal de $8641 \%$ es decir que por cada dólar invertido obtiene 86.41 dólares adicionales, sin embargo si utiliza el Oberón ${ }^{\circledR}$, para el control de ácaro blanco por cada dólar invertido obtendrá una tasa de retorno marginal de $251 \%$ lo que indica un 2.51 dólares recuperados por cada dólar de inversión. El tratamiento Ajo+chile+jabón presenta la mayor tasa de retorno por tener menores costos variables así mismo maneja el ácaro blanco en niveles bajos lo que nos lleva a obtener los segundos mejores rendimientos en el cultivo de chiltoma.

\section{CONCLUSIONES}

El tratamiento que presento mejor efectividad sobre las poblaciones del ácaro blanco fue el Oberón ${ }^{\circledR}$, seguido del Ajo+chile+jabón.

La menor incidencia de daño de $P$. latus lo presentó el tratamiento Oberón $\AA$, seguido de Abamectina ${ }^{\circledR}$ y la menor severidad fue presentada por los tratamientos Oberón ${ }^{\circledR}$ y Ajo+chile+jabón.

Los mayores rendimientos comerciales y los mejores beneficios netos los presentaron el tratamiento Oberón ${ }^{\circledR}$ con $11 \quad 844.62$ USD ha ${ }^{-1}$ seguido de Ajo+chile+jabón con 11279.56 USD ha $^{-1}$.

La mayor tasa de retorno marginal la presentó el tratamiento Ajo+chile+jabón con 8 641\% seguido del tratamiento Oberón con $251 \%$ respectivamente.

\section{AGRADECIMIENTO}

Los autores de esta investigación agradecen a estudiantes de la UNN -Matagalpa Rosa Machado, Luis Carcache, Juan Guido, Robinson Morales y Kevin Morales, al IPADEN-San Isidro donde se realizó la investigación, al Instituto Nicaragüense de tecnología agropecuria (INTA) VI Region por el financiamiento económico de esta investigación.

Cuadro 4. Análisis de la tasa de retorno marginal

\begin{tabular}{|c|c|c|c|c|c|}
\hline Tratamiento & Costo variable & Costo marginal & Beneficio neto & Beneficio marginal & $\begin{array}{c}\text { Tasa marginal de } \\
\text { retorno }(\%)\end{array}$ \\
\hline Testigo & 38.94 & & 6254.56 & & \\
\hline Ajo+chile+jabón & 97.09 & 58.15 & 11279.56 & 5025 & 8641 \\
\hline Oberón $\AA$ & 322.64 & 225.55 & 11844.62 & 565 & 251 \\
\hline
\end{tabular}




\section{REFERENCIAS BIBLIOGRÁFICAS}

Balzarini, MG; Di Rienzo, J. 2012. InfoGen. FCA, Universidad Nacional de Córdoba, Argentina. URL http://www.info-gen.com.ar.

Bolaños, A. 1998. Introducción a la olericultura, editorial universitaria estatal a distancia. San José, CR. 380 p.

CATIE (Centro Agronómico Tropical de Investigación y Enseñanza). 1993. Guía para el manejo integrado de plagas del cultivo del chile dulce. Programa de mejoramiento del cultivo. MIP. Turrialba, CR. 168 p.

CENAGRO (Censo nacional Agropecuario, NI). 2011. Uso de la tierra y distribución del uso de la tierra del sector agropecuario. (en linia) citado el 23-09-2015. www.inide.gob.ni/CENAGRO INFIVCENAGRO/IVCENAGROINFORME

CYMMYT (Centro Internacional para el Mejoramiento de Maíz y Trigo). 1988. La Formulación de recomendaciones a partir de datos económicos. Un manual metodológico de evolución económica. ME. DF. CIMMYT.P.79.

De Coss-Romero, M; Pena, JE. 1998; Relationship of broad mite (Acari: Tarsonemidae) to host phenology and injury levels in (Capsicum annuum, L.). Fla Entomologist. 81(4):515-526.

Di Rienzo, JA; Casanoves, F; Balzarini, MG; González, L; Tablada, M; Robledo, CW. InfoStat versión 2013. Grupo InfoStat, FCA, Universidad Nacional de Córdoba, Argentina. http://www.infostat.com.ar.

FAO (Organización de las Naciones para la Agricultura y la Alimentación). 2004. Base estadística de la FAO. (en linia). Managua, NI. Consultado 6 oct. 2015). Disponible en http//www.fao.org./index-es htm.

González, J; Obregón, H. 2007. Evaluacion de alternativas de protección físicas y químicas de semilleros de chiltoma (Capsicum annum, L.) contra el ataque del complejo mosca blanca (Bemisia tabaci, Gennadius.) - geminivirus. Tesis Ing. Sistemas de protección agrícola y forestal. UNA, Managua, NI. 69 p.

INTA (Instituto Nicaragüense de Tecnología Agropecuaria). 2005. Descriptor de la tecnología No CD-046. Variedad de chiltoma criolla de tres cantos. (en línea). Consultado el 19 sep. 2016. Disponile en www.funica.org.ni/docs/cult_div_46.pdf

2004. Manejo integrado de plagas. Cultivo de el pimiento dulce. Managua, NI. 32 p.

Jiménez-Martínez, E; Barberena, J; Lacayo, Y. 2013. Uso de plaguicidas químicos y botánicos para el manejo de las principales plagas del pimiento dulce (Capsicum annum, L.) en Tisma-Masaya. La Calera 13(20):1-8.

Jiménez-Martínez, E; Martínez, R; Jirón, M. 2013. Plaguicidas botánicos y químicos para el control del ácaro blanco (Polyphagotarsonemus latus, Bank.) (Acarina: Tarsonimidae) en chiltoma (Capsicum annuum, L.), Tisma, Masaya. La Calera 13(20):9-15.

Jiménez-Martínez; E; Varela G. 2013. Módulo práctico: Manejo integrado de plagas. Managua, NI, Universidad Nacional Agraria. 61 p.

King, A; Saunders. 1998. Las plagas invertebradas de cultivos anules alimenticios en América Central. CATIE. Turrialba, CR. 241 p.

Ochoa, R; Aguilar, H; Vargas, C. 1991. Ácaros fitofagos de América Central: guia ilustrada. Serie técnica, manual técnico N 6 . Turrialba, CR. CATIE. Isbn: 9977-57-115-5. Pag 37 A 42. 251 p.

Rayo, I; Mena, A. 2015. Evaluacion de cinco productos botánicos para el manejo del ácaro blanco (Poliphagotarsonemus latus, Bank.) en chiltoma (Capsicum anum, L.) en Tisma-Masaya (Tesis ing) UNA-Managua NI. p 7.

Rodríguez Morell, H; Miranda Cabrera, I; Montoya, A; Rodríguez, Y; Ramos Lima, M. 2012. Comportamiento poblacional de Polyphagotarsonemus latus (Bank) en pimiento (Capsicum annuum, L.), en cultivo protegido. Fitosanidad 12(4):215-220.

Rodríguez, H; Montoya, A; Pérez-Madruga, Y; Ramos, M. 2013. Reproducción masiva de ácaros depredadores Phytoseiidae: retos y perspectivas para Cuba. Protección Vegetal 28(1):12-22.

Rodríguez, H; Montoya, A; Miranda, I; Rodríguez, Y; Depestre, T L; Ramos, M; Zabeh V, MHB. 2015. Biological control of Polyphagotarsonemus latus (Banks) by the predatory mite Amblyseius largoensis (Muma) on sheltered pepper production in Cuba. Protección Vegetal 30(1):70-76.

Santolaria, V. 2013 Actividad enzimática y susceptibilidad a cartap y abamectina en poblaciones de campo de Tuta absoluta (Meyrick) (Lepidóptera: Gelechiidae) (Tesis Ing.) valdivia-Chile. (en línea). Consultado el 13 sep. 2016. Disponible en cybertesis.uach.cl/tesis/ uach/2013/far564a/doc/far564a.pdf

Vanderplank, JE. 1963. Plant, diseases; epidemiology and Control. New York, academy pres. 69 p. 